Reply to "Down-to-Earth" is Imperative! On the Problems of Landscape-Ecological Regionalisation by Means of Multivariate Statistical Methods Friedrich Weller (Ravensburg, Germany) in DIE ERDE 2009, 140:113-125

Replik auf Bodenhaftung tut not! Zur Problematik landschaftsökologischer Regionalisierung mit Hilfe multivariat-statistischer Methoden Friedrich Weller (Ravensburg) in DIE ERDE 2009, 140:113-125

Winfried Schröder*

\title{
Abstract
}

The reply addresses the severe criticism of F. Weller in DIE ERDE 2009, 140:113-125 dealing with the ecological land classification which was first published in Umweltwissenschaften und Schadstoff-Forschung 12 (4), S. 237-243 and later on detailed in the Handbuch der Umweltwissenschaften, 17. Erg.Lfg. 2006. Mainly, the critique concentrates on the following three issues: 1 . The regionalisation dissects uniform landscapes each exhibiting a typical ecological pattern and combines the separated parts with parts of other landscapes to form ecological heterogeneous land classes. 2. The calculation of average values of ecological characters of these heterogeneous land classes is useless and absurd. 3. The nomenclature of the statistically derived land classes violates the correct use of traditional landscape names. The reply shows that, on the one hand, Weller's criticism is due to the impression that he does not understand the statistical method used to calculate the ecological land classification. On the other hand the reply seizes that Weller's idea of land classification does not meet the epistemological principles of experimental sciences such as methodical transparency as well as objectivity and reproducibility of results.

Keywords Ecological land classification; Geographic Information System (GIS); multivariate statistics; objectivity; reproducibility; transparency.

*Correspondence: wschroeder@iuw.uni-vechta.de Lehrstuhl für Landschaftsökologie, Universität Vechta, PF 1553, 49364 Vechta, Deutschland

\section{SpringerOpen ${ }^{\circ}$}

(c) 2011 Schröder; licensee Springer. This is an open access article distributed under the terms of the Creative Commons Attribution License (http://creativecommons.org/licenses/by/2.0), which permits unrestricted use, distribution, and reproduction in any medium, provided the original work is properly cited. 


\begin{abstract}
Zusammenfassung
Die Replik gilt der scharfen Kritik von F. Weller in DIE ERDE 2009, 140:113-125 an der landschaftsökologischen Raumgliederung. Diese, erstmals veröffentlicht in Umweltwissenschaften und Schadstoff-Forschung 12 (4), S. 237243, und ausführlich behandelt im Handbuch der Umweltwissenschaften, 17. Erg.Lfg. 2006, habe, so Weller, im Wesentlichen folgende Mängel: 1. Die Gliederung zerschneide einheitliche Landschaften, die durch ein jeweils typisches ökologisches Gefügemuster geprägt seien, und setze diese Raumeinheiten mit solchen anderer Landschaften zu heterogenen Raumklassen zusammen. 2. Die Berechnung von Mittelwerten der Ausprägungen ökologischer Merkmale dieser inhomogenen Raumklassen sei unbrauchbar. 3. Die Benennung dieser inhomogenen Raumeinheiten beruhe auf der falschen Verwendung z.T. traditioneller Landschaftsnamen. In der Replik wird gezeigt, dass Wellers Kritik zum einen darauf beruht, dass er das der landschaftsökologischen Raumgliederung zugrunde liegende statistische Verfahren nicht verstanden hat. Zum anderen wird dargelegt, dass Wellers Gegenmodell grundlegenden wissenschaftlichen Kriterien wie Transparenz des Verfahrens sowie Bearbeiterunabhängigkeit (Objektivität) und Reproduzierbarkeit des Ergebnisses nicht entspricht.
\end{abstract}

Schlagwörter Geografisches Informationssystem (GIS); Landschaftsökologische Raumgliederung; multivariate Statistik; Naturraumgliederung; Objektivität; Reproduzierbarkeit; Tansparenz.

Aus einer Umweltbehörde, die wie auch andere die erstmals im Jahr 2000 veröffentlichte landschaftsökologische Raumgliederung Deutschlands [1] verwendet, wurde ich kürzlich auf F. Wellers Gedanken Zur Problematik landschaftsökologischer Regionalisierung mit Hilfe multivariat-statistischer Methoden hingewiesen. Anlass für die Kritik Wellers war im Jahr 2006 die Veröffentlichung der methodologischen Grundlagen der landschaftsökologischen Raumgliederung Deutschlands und regionaler Spezifikationen im Handbuch der Umweltwissenschaften [2].

Ich begrüße die Wiederaufnahme einer lange zurückliegenden Methodendiskussion mit Herrn Kollegen Weller. Eine solche Diskussion hatte ich Herrn Weller zuletzt nach einem intensiven Austausch 2006 / 2007 über Verfahren der Raumgliederung vorgeschlagen. Ich regte an, unsere kontroversen Standpunkte im Handbuch der Umweltwissenschaften oder in der bei den Nutzern der landschaftsökologischen Raumgliederung gelesenen Umweltwissenschaften und Schadstoff-Forschung (UWSF; seit 2010: Environmental Science Europe - ESEU) einzubringen. Diesen Vorschlag hatte ich mit der Bitte verknüpft, dass er oder das jeweilige Veröffentlichungsorgan mich informiert, wenn meinem Vorschlag gefolgt werden konnte. Einen entsprechenden Hinweis habe ich nicht erhalten. Da das inhaltliche Portfolio der ERDE mit meinen Arbeitsschwerpunkten nur kleine Überschneidungen aufweist, ich sie infolgedessen nicht lese und deshalb den Beitrag Wellers nicht bemerkte, erfolgt meine Reaktion so spät.

Dass ich eine Replik auf F. Wellers Kritik überhaupt verfasse, ist eher dem Respekt vor dem Kritiker selbst und der Einsicht in die Notwendigkeit einer Methodendiskussion geschuldet als dem Bedürfnis, das eigene Vorgehen zu verteidigen. Diese Replik auf F. Weller ist auch Ausdruck meines Bedürfnisses, diejenigen zu entlasten, die die Arbeit an der landschaftsökologischen Raumgliederung konstruktiv methodenkritisch begleiteten und von denen F. Weller nun den einen oder anderen als Anwalt seiner Kritik anführt. Dazu zählen Gutachter meiner Habilitationsschrift. Ferner sind es Gutachter der Aufsätze, in denen die Raumgliederung bislang behandelt wurde - einschließlich des von F. Weller in den Mittelpunkt seiner Kritik gestellten Handbuchartikels. Und es sind diejenigen, die als wissenschaftliche Beiräte der Projekte, in denen die Raumgliederung entwickelt wurde, tätig waren und über Jahre mit großem Fachverstand immer wieder die "dumme Frage des Außenstehenden" (Weller, S. 120) stellten. Dasselbe gilt auch für den Arbeitskreis Geoökologische Kartierung und Leistungsvermögen des Landschaftshaushaltes der Deutschen Akademie für Landeskunde, mit dem die landschaftsökologische Raumgliederung in fachlichem Einvernehmen diskutiert wurde. Schließlich sind es diejenigen, die die Förderung von Projekten durch die Deutsche Forschungsgemeinschaft ermöglichten, die auf der landschaftsökologischen Raumgliederung aufbauten.

Die von F. Weller kritisierte landschaftsökologische Raumgliederung Deutschlands wurde wie folgt berechnet: Das Gebiet wurde in rund 88.400 Zellen à $2 \mathrm{~km} \times 2 \mathrm{~km}$ untergliedert. Jede von ihnen wird anhand der in 67 Klassen differenzierten potenziell natürlichen Vegetation (kategoriale Ziel- / Kriteriumsvariable), Bodenart (25 Kategorien), Klima (Niederschlag, Lufttemperatur, Verdunstung Januar bis Dezember sowie Globalstrahlung März bis November) und Höhenlage (metrisch) quantitativ beschrieben. Jedes dieser 48 Merkmale ging als GIS-Karten in die Berechnung ein. Die o.g. Rasterung der Karten orientiert sich an der geringsten bekannten räumlichen Auflösung der ihnen zugrunde liegenden Daten. Mit Classification and Regression Trees (CART) wurden die 48 mal 88.4000 Rasterzellen nach dem Grade 
ihrer Ähnlichkeit in Bezug auf die Ausprägung der genannten landschaftsökologischen Merkmale klassifiziert. Die gebildeten Raumklassen unterscheiden sich in Bezug auf die sie kennzeichnenden Merkmale statistisch signifikant bei gleichzeitig optimierter klasseninterner Homogenität. Das Maß der erreichbaren Homogenität hängt dabei ab von der Klassenanzahl: Je höher die Zahl der Raumklassen, desto homogener sind diese. Dieser Zusammenhang wird theoretisch erklärt und am Beispiel Norddeutschlands mit Kartenbeispielen veranschaulicht.

Die Kritik F. Wellers an dieser landschaftsökologischen Raumgliederung „resultiert im Wesentlichen aus drei Beobachtungen: 1. Die Gliederung zerschneidet einheitliche Landschaften mit einem jeweils typischen ökologischen Gefügemuster und setzt die Teile mit Teilen anders strukturierter Landschaften zu völlig heterogenen Raum- oder Ökoklassen zusammen. 2. Die Berechnung von Mittelwerten ökologisch relevanter Faktoren für diese heterogenen Gebilde ist wenig hilfreich, da sie für keinen der Teile eine brauchbare Aussage ergibt. 3. Bei der Benennung der derart abgegrenzten Raumeinheiten werden z.T. traditionelle Landschaftsbezeichnungen falsch verwendet" (S. 115).

Um den Kern meiner Replik vorweg zu nehmen: F. Weller lässt an keiner Stelle seiner Kritik erkennen, wie die von ihm als Referenzsysteme fachlich richtiger Raumgliederung favorisierten Ansätze die Einheitlichkeit von Landschaften und das Ausmaß, wie typisch ihre ökologischen Gefügemuster sind, operationalisieren. $\mathrm{Zu}$ insinuieren, die beteiligten Fachleute und die Autoren des inkriminierten Handbuchartikels zur landschaftsökologischen Raumgliederung hätten sich daran beteiligt, ein mit Computerspielen vergleichbares Instrument (Weller, S. 121) ermöglicht bzw. entwickelt zu haben, ist wenig zweckdienlich. Wissenschaft braucht Kritik, aus der klar ersichtlich wird, wie vorhandene Schwächen durch Methodenentwicklung vermindert werden können. Diese Gelegenheit hat F. Weller nicht genutzt. Er teilt im Kern lediglich mit, dass die Raumklassen der landschaftsökologischen Gliederung nicht hinreichend mit denjenigen übereinstimmten, die mit „Bodenhaftung“ (passim) und "primärer sinnlicher Erfahrung" (S. 120) angefertigt wurden. Wie diese allerdings entstanden sind, berichtet er nicht. Analysiert man die entsprechenden Arbeiten, erkennt man schnell, dass sie keine für eine Reproduktion der Ergebnisse hinreichende Methodenbeschreibung enthalten. Genau dies jedoch ist eines der zentralen Kriterien empirischer Wissenschaften für eine methodologisch stringent durchgeführte Untersuchung: Ihre Ergebnisse müssen sich von allen hinreichend ausgebildeten Personen, also unabhängig vom forschenden Subjekt, in Wiederholungen erzielen lassen.

Neben Reproduzierbarkeit und Objektivität ist die Validität das dritte Kriterium, an dem sich Ergebnisse empirischer Forschung messen lassen muss. In Untersuchungen wie in der von F. Weller inkriminierten bemisst sich die Validität nach der Qualität der verwendeten Daten und nach der Geeignetheit des verwendeten Verfahrens, mit dem die Daten ausgewertet werden. Die für die landschaftsökologische Raumgliederung verwendeten Daten wurden von Fachbehörden des Bundes nach einschlägigen QA-Richtlinien flächendeckend mit jeweils bundesweit einheitlicher Methodik erhoben. An deren Auswahl haben zahlreiche Fachleute aus wissenschaftlichen Oberbehörden des Bundes und den entsprechenden Dienststellen der Bundesländer mitgewirkt. Das eingesetzte statistische Verfahren wurde auf Grundlage internationaler Fachpublikationen als leistungsfähig und dem Datenmaterial angemessen eingestuft. Wenn die Ergebnisse der auf dieser Daten- und Methodengrundlage berechneten Raumgliederung nach F. Wellers Einschätzung - anhand welcher Methoden ist er zu dieser gekommen? - mit keiner Gliederung, die mit „Bodenhaftung“ (passim) und "primärer sinnlicher Erfahrung" (S. 121) gewonnen wurden, hinreichend übereinstimmen (S. 119), so müsste man sich zunächst der Entstehung der zuletzt genannten Befunde detailliert widmen. Anschließend wäre das Ausmaß an Übereinstimmung / Abweichung quantitativ zu bestimmen. Doch F. Weller gibt keinerlei Hinweise, ob und wie dies konkret geschehen könnte.

Die mangelnde Übereinstimmung einzelner Klassen der landschaftsökologischen Raumgliederung mit den durch die Methode "Bodenhaftung“ erzielten Raumklassen nimmt in nur leicht variierten Spielarten den Hauptteil der Kritik F. Wellers ein (S. 115 -118). Dabei kommt ihm sicherlich ein aus meiner Sicht ggf. erklärungsbedürftiges Charakteristikum der kritisierten landschaftsökologischen Raumgliederung entgegen. Dieser besteht darin, dass für die Klassen der landschaftsökologischen Raumgliederung "traditionelle Landschaftsbezeichnungen" (S. 115) verwendet wurden. Sie wurden in Analogie zu den Usancen z.B. in der Geologie nach dem Schwerpunkt ihrer geografischen Verbreitung in den Klassen der naturräumlichen Gliederung [3] benannt. In Erwartung dadurch entstehenden Erklärungsbedarfs hatte sich der Verfasser zunächst gegen eine Namensgebung gewendet und für eine bloß numerische Bezeichnung plädiert. Doch das Argument, jemand würde eine geologische Karte sicherlich nicht allein deshalb anzweifeln, wenn sie das Perm auch in Gegenden außerhalb des onomasiologisch maßgeblichen ehemaligen russischen Gouvernements Perm am Fuße des Uralgebirges ausweist, überzeugte mich schließlich.

Eine umfassende wissenschaftstheoretische Auseinandersetzung mit der Kritik F. Wellers soll an dieser Stelle unterbleiben. Denn zum einen sind die methodologischen Grundlagen der landschaftsökologischen Raumgliederung 
ausführlich veröffentlicht. Und zum anderen setzte eine solche Debatte voraus, dass zunächst einmal die Ergebnisse der Raumgliederungen mit "Bodenhaftung“ (passim) und „primärer sinnlicher Erfahrung“ (S. 121) methodisch konkretisiert abgeleitet werden. Daher möchte ich meine Replik mit einigen Anmerkungen zu einigen weiteren Kritikpunkten F. Wellers zunächst einmal beschließen.

- Beginnen möchte ich dabei mit den einleitenden Bemerkungen der Herausgeber. Diese führen aus: „Mit seinen Kritikpunkten, Warnungen und Postulaten steht Weller nicht allein", und sie befürchten, dass sich die - dabei? - „... abzeichnende Dichotomie ... einer sachorientierten, methodisch weiterführenden konstruktiven Auseinandersetzung im Wege steht." Vorsichtig fügen sie an, ihre "Aufforderung zur sachlichen Auseinandersetzung sollte nicht a priori als Angriff auf notwendige neue methodische Ansätze missverstanden werden.

Wenn die Kritik F. Wellers ein Beispiel aus einer breiten Kritikerfront sein sollte, dann sollte diese auch zur Darstellung gelangen. Dies kann der Methodenentwicklung dienlich sein, wenn es sich um operationalisierungsfähige Kritik handelt. Genügt die Auseinandersetzung diesem Kriterium, so steht auch eine Dichotomie einer fruchtbaren Auseinandersetzung nicht im Wege.

- Für F. Weller verstelle die „vorrangige Beschäftigung mit diesen Hilfsmitteln vielfach der Blick auf die eigentlichen Objekte“ (S. 114), wobei "Hilfsmittel“ die Anwendung von statistischen Verfahren und GIS meint. Grund dafür sei zum einen, „dass Ergebnisse früherer Untersuchungen und Erhebungen, soweit sie nicht in digitaler Form vorliegen, für den Bearbeiter gar nicht existieren; zum anderen - und das wiegt schwerer - fehlt ihm oft der unmittelbare sinnliche Kontakt zu den von ihm bearbeiteten Objekten."

F. Weller hat Recht, wenn er die empirische Erdung statistischer und dynamischer Modelle fordert. Doch ist es unrealistisch zu erwarten, dass jeder Landschaftsökologe deutschlandweit über flächendeckenden sinnlichen Kontakt und daraus abgeleitete Daten verfügt, deren methodische Genese den Kriterien der Objektivität, Reliabilität (Reproduzierbarkeit) und Validität hinreichend entspricht. So wie Herr Weller für sich reklamiert, Baden-Württemberg besonders gut zu kennen, so könnte der Verfasser dies beispielsweise für Schleswig-Holstein für sich in Anspruch nehmen. Eine landschaftsökologische Gliederung, die Flächengrößen erreicht, die von einzelnen Forschern unmöglich durch eigene Erhebungen in Forschungsprojekten bewältigt werden können, wie beispielsweise das Gebiet Deutschlands oder Europas, ist auf die Verwendung von Daten verwiesen, die dafür autorisierte Institutionen nach einschlägigen methodischen Richtlinien erheben. Genau dies wurde in dem von F. Weller kritisierten Fall getan. Im Übrigen: Es gibt halt Phänomene, die sich dem sinnlichen Kontakt der Menschen hartnäckig entziehen. Oder hat jemals ein Landschaftsökologe Protonen in der Bodenlösung oder den Jahresgang des Niederschlags in der letzten vollständigen Klimanormalperiode quantifizierbar sinnlich erfahren? Hat es ein Landschaftsökologe bislang vermocht, durch primäre Sinneserfahrung festzustellen, welche der rund 88.400 , jeweils $2 \mathrm{~km}$ mal $2 \mathrm{~km}$ großen Rasterzellen Deutschlands sich in Bezug auf den Niederschlag oder die Ausprägung eines der 47 weiteren, für die Raumgliederung verwendeten landschaftsökologischen Merkmale sich hinreichend ähnlich sind und deshalb in einer Klasse zusammengefasst werden können? Konnte ein Landschaftsökologe jemals sinnlich erfahren, welche der zahlreichen Merkmalsausprägungen statistisch am aussagekräftigsten für die Klassenbildung ist?

Diese weiter ausbaubaren Fragen lassen erkennen, dass nichts damit gewonnen ist, die Richtigkeit der statistisch abgeleiteten landschaftsökologischen Raumgliederung an Forschungsergebnissen eines Landschaftsökologen zu messen, deren Grundlage der „unmittelbare sinnliche Kontakt zu den von ihm bearbeiteten Objekten" (S. 114) ist. Somit setze ich mich im Folgenden mit F. Wellers Gedanken auseinander, die er seinen ausführlichen Schilderungen der Abweichungen zwischen der landschaftsökologische Raumgliederung und den durch sinnlichen Kontakt und Bodenhaftung gewonnenen Erkenntnissen ab Seite 119 anschließt.

- F. Weller kritisiert, dass „gravierende[ ] ökologische[ ] Unterschiede innerhalb einer ... Raumklasse völlig außer Betracht" bleiben. Dabei übersieht er, dass die Homogenität / Heterogenität der Klassen der landschaftsökologischen Raumgliederung statistisch operationalisiert und mit entsprechenden Maßen quantifiziert wird. Ermöglicht dies sein Gegenentwurf, die nicht näher beschriebene Methode der sinnlichen Erfahrung und Bodenhaftung? Natürlich sind die Klassen der landschaftsökologischen Raumgliederung nicht völlig homogen. Wären sie dies, dann bräuchten wir wohl fast so viele Klassen wie zu klassifizierende Objekte - das sind hier die $2 \mathrm{~km}$ mal $2 \mathrm{~km}$ großen Rasterzellen. Eine im jeweiligen Verwendungszusammenhang handhabbare Klassifizierung sollte möglichst wenige Klassen besitzen. Der dafür zu zahlende Preis ist in der Regel eine höhere klasseninterne Heterogenität. Diese ist nur scheinbar gleich Null, wenn wir jeden geografisch exakt definiert Ort als Unikat und mit "primärer, sinnlicher Erfahrung" (S. 120) und 
"Bodenhaftung" (passim) betrachten. Doch der Landschaftsökologe, der sich der Grenzen seiner sinnlichen Sensibilität bewusst ist und deswegen diese durch Nutzung sensiblerer Messgeräte erhöht, wird die dem makroskopisch homogenen Objekt innewohnende Heterogenität erkennen. Was nun?

Die Antwort auf Fragen nach dem Ausmaß von Homogenität und Heterogenität hängt $a b$ von der räumlichen und zeitlichen Auflösung der betrachteten Objekte sowie von dem das tolerable Ausmaß von Homogenität und Heterogenität bestimmenden Verwendungszusammenhang entsprechender Befunde. Im Falle der landschaftsökologischen Raumgliederung in 21 Klassen entspricht die Klassenzahl ihrem Verwendungszweck, der ökologischen Flächenstichprobe für die umweltökonomischen Gesamtrechungen des Statistischen Bundesamtes, das durch Experten im wissenschaftlichen Beratergremium der Raumgliederungsprojekte mitwirkte. Die mit den seinerzeit verfügbaren, Deutschland flächendeckend abbildenden Daten maximal mögliche Differenzierung der landschaftsökologischen Raumgliederung in 73 Stufen löst viele der von $\mathrm{F}$. Weller kritisierten vermeintlichen Abweichungen der mit „unmittelbare[m] sinnliche[n] Kontakt" (S. 114) und Bodenhaftung (passim) erfassten "Lebensraumwirklichkeit" (S. 121) auf.

- F. Weller ist überzeugt, „dass diese Raumgliederung am Computer entwickelte virtuelle Landschaften abbildet, die die ökologische Situation Deutschlands höchst unangemessen wiedergeben. Völlig unverständlich ist, warum die Autoren offensichtlich nie auf die Idee gekommen sind, ihre Raumgliederung wenigstens stichprobenartig mit den realen Verhältnissen zu vergleichen. Man kann sich des Eindrucks nicht erwehren, dass hier über der Konzentration auf die ausschließlich von dritter Seite gelieferten Daten und deren technischer Verarbeitung der Bezug zur konkreten Landschaft schlicht verloren gegangen ist (falls er überhaupt je bestanden hat). Andernfalls hätte den Autoren die Diskrepanz zwischen ihrer Raumgliederung und der tatsächlichen Situation sehr schnell auffallen müssen - allein schon beim Blick auf Relief und Vegetation" (119).

F. Wellers Kritik wirkt erdrückend, zeigt jedoch nicht auf, wie Besserung herbeigeführt werden könnte. Hierzu bedürfte es zunächst einmal der Klärung, was er unter „ökologische Situation Deutschlands“, unter „konkrete Landschaft" und unter "tatsächliche Situation" (alle drei Zitate S. 119) versteht und wie er diese und die Angemessenheit ihrer Erfassung reproduzierbar, objektiv und valide $\mathrm{zu}$ dokumentieren gedenkt. Oder gelten diese allgemein anerkannten Kriterien nicht für die von ihm propagierte Methodik? Weller suggeriert, dass die von ihm auf S. 119 aufgeführten, durch „primäre[ ] sinnliche Erfahrung“ (S. 120) und „Bodenhaftung“ gewonnenen Raumgliederungen erfassten evidenterweise das, was er zuvor als „ökologische Situation Deutschlands“, als „konkrete Landschaft", als "tatsächliche Situation“ (S.119) oder später als „reale Landschaft" und „Lebensraumwirklichkeit" (S. 121) bezeichnet.

Seine im Prinzip sinnvolle Forderung nach Erfassung der „ökologische[n] Situation Deutschlands“ läuft letztlich darauf hinaus, das 1978 von Ellenberg, Fränzle und Müller entworfene Konzept für ein ökologisches Informationssystem zu verwirklichen. Dass dies in der damals beabsichtigten Stringenz bis heute nicht gelungen ist, wirft ein interessantes Schlaglicht auf die Forderungen Wellers: Es ist gewagt zu insinuieren, er oder die Autoren der von ihm als Referenz für Richtigkeit herangezogenen Raumgliederungen verfügten über die Kenntnisse und Daten, die ökologische Situation Deutschlands angemessen darstellen zu können. Umso mehr stellt sich diese Frage den Geowissenschaftlern dort, wo ohne technische Hilfsmittel keine landschaftsökologisch orientierte Stratifizierung eines Untersuchungsraums vorgenommen werden kann: am Meeresgrund. Auch hier werden räumliche Klassifikationssysteme benötigt, um physischgeographische Gegebenheiten ausreichend repräsentativ abzubilden. Letzteres kann aber nur unter Verwendung vorhandener Daten und der Anwendung objektiver Methoden nachvollziehbar erfolgen.

Ich hoffe, dass der Methodenstreit zwischen F. Weller und mir ein wenig dazu beiträgt, dass bald bessere als die bisher vorliegenden Raumgliederungen entwickelt werden. Auf Grundlage des von F. Weller kritisierten Ansatzes haben wir mit Förderung der DFG eine europäische Raumgliederung berechnet und einen in den Meeresumweltwissenschaften international auch von Empirikern anerkannten, vom BMBF geförderten Vorstoß zur Kartierung mariner Habitate unternommen. Dieser soll in einem langfristig angelegten Projekt ausgebaut werden - durch Kooperation biologischer und geologischer Empirie sowie Statistik / Geoinformatik. Hierbei und darüber hinaus wird sich die Landschaftsforschung auf neue Herausforderungen einstellen müssen, die sich nicht durch „primäre sinnliche Erfahrung mit Bodenhaftung" (Weller, passim) bewältigen lassen dürfte und hier nur beispielhaft und stichwortartig benannt werden können: Integration verteilter Daten; bessere remote sensing-Auflösungen und deren Auswertbarkeit; Erfordernisse, die Empfindlichkeit von Räumen für Eingriffe in einem zunehmend durch vereinheitlichte (Genehmigungs-) Standards gekennzeichneten Rahmen abzuschätzen (Tendenz zu europaweiten einheitlichen Zulassungen von GVO, NanoMaterialien mit unbekannten Umweltfolgen); repräsentatives EU-weites Monitoring. Hierbei eine 
großräumige Perspektive einzunehmen und quantitativ $\mathrm{zu}$ operationalisieren, die das Potenzial des einzelnen Wissenschaftlers überschreitet und national wie international umweltrelevante aussagekräftige Informationen integriert, ist eine methodologisch zu leistende Notwendigkeit, bei gegebenem technischen und wirtschaftlichen Entwicklungsstand die Nachhaltigkeit, Nutzbarkeit und Diversität der Naturräume bestmöglich zu erhalten.

Received: 01 June 2011 Accepted: 08 June 2011

Published: 08 June 2011

\section{Literatur}

1. Schröder W, Schmidt G: Raumgliederung für die Ökologische Umweltbeobachtung des Bundes und der Länder. Umweltwiss Schadst Forsch 2000, 12:236-243.
2. Schröder W, Schmidt G, Hornsmann I: Landschaftsökologische Raumgliederung Deutschlands. In Handbuch der Umweltwissenschaften. Grundlagen und Anwendungen der Ökosystemforschung. Kap. V-1.9, 17. Erg.Lfg Hrsg. Fränzle O, Müller F, Schröder W. Landsberg am Lech, München, Zürich, 2006:1-100.

3. Meynen E (Hrsg.): Handbuch der naturräumlichen Gliederung

Deutschlands. Selbstverlag der Bundesanstalt für Landeskunde. 2 Bde. Remagen / Bad Godesberg 1959-1962.

doi:10.1186/2190-4715-23-20

Cite this article as: Schröder W: Reply to "Down-to-Earth" is Imperative! On the Problems of Landscape-Ecological Regionalisation by Means of

Multivariate Statistical Methods. Friedrich Weller (Ravensburg, Germany) in DIE ERDE 2009, 140:113-125. Environmental Sciences Europe 2011, 23:20. 